\title{
VSR Sort: A Novel Vectorised Sorting Algorithm \& Architecture Extensions for Future Microprocessors
}

\author{
Timothy Hayes* ${ }^{* \dagger}$, Oscar Palomar* ${ }^{* \dagger}$, Osman Unsal*, Adrian Cristal ${ }^{* \dagger \dagger}$ and Mateo Valero*† \\ * Barcelona Supercomputing Center ${ }^{\dagger}$ Universitat Politècnica de Catalunya \\ $\ddagger$ Consejo Superior de Investigaciones Científicas (IIIA-CSIC) \\ $\{$ first $\} .\{$ last $\} @$ bsc.es
}

\begin{abstract}
Sorting is a widely studied problem in computer science and an elementary building block in many of its subfields. There are several known techniques to vectorise and accelerate a handful of sorting algorithms by using single instruction-multiple data (SIMD) instructions. It is expected that the widths and capabilities of SIMD support will improve dramatically in future microprocessor generations and it is not yet clear whether or not these sorting algorithms will be suitable or optimal when executed on them. This work extrapolates the level of SIMD support in future microprocessors and evaluates these algorithms using a simulation framework. The scalability, strengths and weaknesses of each algorithm are experimentally derived. We then propose VSR sort, our own novel vectorised non-comparative sorting algorithm based on radix sort. To facilitate the execution of this algorithm we define two new SIMD instructions and propose a complementary hardware structure for their execution. Our results show that VSR sort has maximum speedups between $14.9 x$ and 20.6x over a scalar baseline and an average speedup of $3.4 x$ over the next-best vectorised sorting algorithm.
\end{abstract}

\section{INTRODUCTION}

Sorting is a widely studied problem in computer science and an elementary building block in many of its subfields including scientific computing and database management systems. There are many unique ways to achieve sorted output and each technique has its own particular strengths and weaknesses. Numerous works have successfully leveraged datalevel parallelism (DLP), when available, to accelerate sorting algorithms [1]-[7].

Recent developments in microprocessor architectures have pushed a focus on multi-core acceleration. While this is an effective technique to exploit thread-level parallelism (TLP), SIMD instruction sets offer a more efficient way to accelerate DLP [8], [9]. There has typically been some level of support for SIMD instructions in general-purpose microprocessors, commonly dubbed multimedia extensions, e.g. MAX-1, Altivec and SSE. Although these ISA extensions started out relatively simple, successive generations have become more sophisticated and offer wider SIMD registers to process more elements per instruction as well as more intricate instructions to operate on them. AVX-512 [10] will increase the width of the registers to 512 bits and include mask registers, full gather/scatter support and many non-trivial SIMD instructions; initial implementations are expected in 2015 for Knights Landing. This trend is anticipated to continue in the future and the SIMD register width and instruction sets are expected to grow further. We therefore predict that the SIMD support found in commodity microprocessors will eventually resemble the instruction sets of classic vector architectures traditionally found in supercomputers [11]. As current SIMD support is still quite restrictive and the transformation from multimedia extensions to true vector support is still incomplete, the true potential of exploiting the DLP found in sorting algorithms is hitherto unknown.

This work makes three principal contributions. (1) We first study prior DLP-accelerated sorting proposals with a uniform and modern platform and assess them using consistent metrics thus allowing us to identify and compare the strengths and weaknesses of each algorithm. In particular, we use an anticipated commodity microprocessor with a sophisticated level of SIMD support to explore vectorised implementations of quicksort, bitonic mergesort and radix sort. (2) Based on these evaluations, we propose a novel non-comparative sorting algorithm: VSR sort is a highly efficient vectorised implementation of radix sort that overcomes many of the drawbacks found in the evaluated sorting algorithms. (3) To facilitate this algorithm, we propose two new instructions as well as a hardware implementation that includes both a serial and parallel variant. Additionally, we suggest several other uses for the new instructions and hardware.

Based on experiments, we report that our VSR sort outperforms the aforementioned prior work and shows good scalability for large SIMD widths. Furthermore, it exhibits good performance using both a simple single-lane pipelined mechanism as well as with a more sophisticated implementation using lockstepped parallel lanes. We show that VSR sort has maximum speedups over a scalar baseline between 14.9x and 20.6x. On average it performs 3.4x better than the next-best vectorised sorting algorithm when run on the same hardware configuration.

Section II discusses the state of the art in DLP acceleration, the parameters we explore and describes our custom simulation framework. Section III outlines the experimental methodology and evaluates three sorting algorithms that leverage DLP. Section IV describes VSR sort. We propose to implement this algorithm by extending the baseline SIMD ISA with two new instructions and recommend two different ways of realising these instructions in hardware. The algorithm is then evaluated and compared with those of Section III. Related work is discussed in Section V. Finally Section VI concludes the article. 


\section{MOdELling ForTHCOMING SIMD SUPPORT}

Here we discuss the DLP parameters relevant to our evaluations. In this work we use the term multimedia extension to mean the current level of SIMD support found in existing microprocessors and the term vector to represent our anticipated level of SIMD support in future microprocessors. The term SIMD is used to encapsulate both the former and the latter and vectorise is defined as the process of transforming an algorithm with DLP to use SIMD instructions. Many microprocessor ISAs have some SIMD support but the state of the art is best represented by Intel's x 86 due to its advances with AVX2, the Xeon Phi and their proposal of AVX-512. This discussion thus limits itself to the DLP facilities found in $\mathrm{x} 86$ microprocessors.

The SIMD capabilities in x86-based microprocessors have moved from simple 64-bit multimedia extensions in MMX to 128 bits in SSE, and to registers of 256 bits in AVX. This width will be enlarged to 512 bits in 2015 with plans to expand to 1,024 bits in future generations [12]. This trend is expected to continue for the foreseeable future thus it is imperative to understand what the performance consequences will be when running vectorisable algorithms on future microprocessors with wider SIMD registers. This work aims to explore the performance characteristics of DLP-accelerated sorting algorithms when the SIMD register width is as large as 2,048 bits.

In x86 architectures, SIMD instructions are issued to functional units that are as wide as the SIMD registers, i.e. all elements of the operation are processed in parallel. This is in contrast to a traditional vector architecture like the CRAY1 [13] which instead pipelined its SIMD instructions. Recent work on vector architectures [14] has shown that there are algorithms that can be accelerated with SIMD instructions that are implemented in a pipelined way yet show little extra benefit when using lockstepped parallel lanes. It is therefore important to be able to experiment with this parallel factor in order to better understand the algorithms' characteristics.

The Xeon Phi offers a more sophisticated SIMD ISA than SSE/AVX and includes useful instructions such as gather/scatter as well as masked operations. One drawback is that its microarchitecture uses a simple dual-issue in-order pipeline based on the original Pentium [15], [16]. Prior work [14], [17] shows that there are advantages when executing SIMD instructions with an out-of-order $(\mathrm{OoO})$ superscalar microarchitecture. It is also felt that to make a fair comparison to scalar algorithms a similar, if not equal, baseline microarchitecture should be used.

We are mindful that there exist other means to exploit DLP; sorting performance has also been improved with dedicated devices like FPGAs [18]-[20] and GPUs [21]-[23]. It is possible that using these accelerators could achieve results more favourable than those presented in this work however it would not be correct to make a direct comparison for two reasons. (1) FPGAs and GPUs haven't strictly superseded SIMD extensions and there are still advantages with tightly integrated DLP support in OoO superscalar processors [24], especially when it is preferable not to offload to an external device [25] or when there is a fine-grained mixture of scalar and vectorisable code, e.g. in DBMSs [14], [26]. (2) Our work evaluates the relative gains of SIMD acceleration within a single core. There has been a lot of work done accelerating sorting with TLP [3], [5], [27], [28] including the algorithms examined in this work [1], [7], [29]. The works of [30] and [31] show that TLP on multicore performs comparably with GPUs when the code is optimised for the platform. The purpose of our research is to uncover DLP bottlenecks within the core itself, nevertheless, TLP and DLP are not mutually exclusive and there is no reason to believe TLP could not be leveraged as well however this is beyond the scope of the article. Our work evaluates sorting with a level of SIMD support expected in the future, and this is important because we have found significant bottlenecks that occur in the SIMD unit itself due to the irregularity of the DLP.

\section{A. Simulation Framework}

Our goals are to look at the performance characteristics of existing vectorised sorting algorithms running on future microarchitectures as well as proposing new instructions and hardware to facilitate innovative algorithms. Achieving these goals would be impossible if using only existing architectures therefore we have created a simulation environment in order to make the necessary experiments.

At its heart we use PTLsim [32], a cycle-accurate x8664 simulator. PTLsim models many features of modern OoO superscalar processors including: $\mu$ op translation, multistage pipelines, speculation and recovery, and a multi-tiered cache hierarchy. We have configured the simulator to behave as close as possible to Intel's Westmere microarchitecture [33]. Table I contains the various microarchitectural parameters. There are six execution unit clusters in total: a load address generation cluster; a store address generation cluster; a store data cluster; and three arithmetic (non-memory) clusters.

TABLE I. MicRoARChITECTURE PARAMETERS

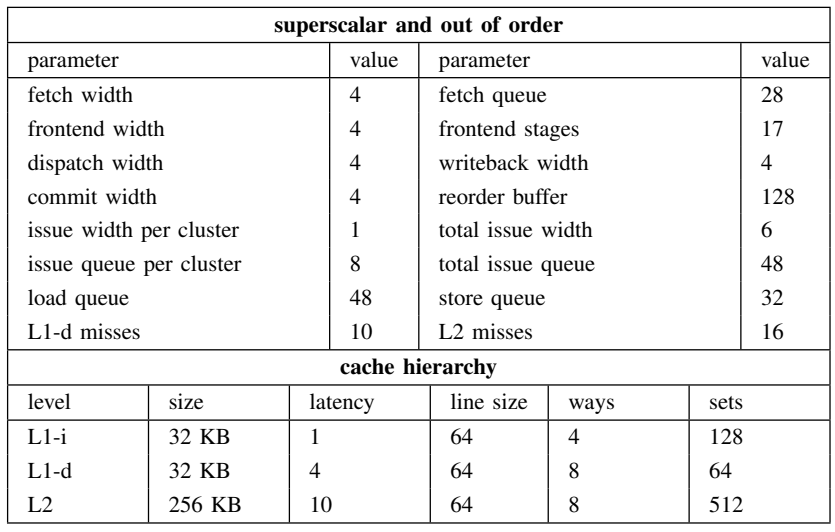

By default PTLsim uses a fixed latency memory system which does not model bandwidth and contention issues. Recent work on vector processors [14] has shown that they have the ability to saturate a system's available bandwidth, thus making it crucial to model the memory system accurately when executing vectorised algorithms, otherwise the results may be inaccurate and misleading. For this reason we have integrated DRAMSim2 [34], a cycle-accurate memory system simulator, into PTLsim and replaced the default memory model. Having an accurate memory model allows the vectorised algorithms to work within a realistic bandwidth envelope and thus enforces a 
fairer comparison to non-vectorised algorithms. Table II shows the memory system parameters. The simulated processor has a frequency of $2.67 \mathrm{GHz}$ and as a result the memory controller is clocked every four processor cycles. Additionally, the following address layout scheme is used as it was found to work well with all of our experiments: row:rank:bank:column:burst.

TABLE II. MEMory System Parameters

\begin{tabular}{|l|l|l|l|}
\hline parameter & value & parameter & value \\
\hline type & DDR3-1333 & transaction queue & 64 \\
clock & $1.5 \mathrm{~ns}$ & command queue & 256 \\
policy & open page & row accesses & 8 \\
queue & per rank per bank & banks & 8 \\
scheduling & rank then bank & ranks & 4 \\
rows & 32,768 & columns & 2,048 \\
burst length & 64 bytes & device width & 4 \\
\hline
\end{tabular}

1) SIMD Support: We have modified the simulation framework substantially in order to give it extensive SIMD support. For brevity, we give a high-level overview that captures the most important features of these SIMD additions.

We have extended the $\mathrm{x} 86-64$ ISA with sixteen logical vector registers and four logical mask registers. The width of these registers is a configurable parameter of the simulator in order to experiment with different maximum vector lengths (MVL). As the baseline microarchitecture uses register renaming, we also apply this technique to the new vector registers. It has been shown that renaming vector registers is beneficial when the number of physical registers is double the number of logical registers [17] hence we provide thirty-two physical vector registers and eight physical mask registers. There is also an additional vector length register that controls the number of elements operated on by any given vector instruction. This is explicitly managed with get/set instructions.

The vector capabilities are tightly integrated into the microarchitecture. We have added three new clusters: one to perform the address generation of vector memory instructions and two to execute non-memory vector instructions.

We have defined and implemented new vector memory instructions. Three patterns are supported, each with its own load, store and prefetch instructions: (1) unit-stride: memory is accessed contiguously. This is the most efficient access pattern due to the spatial locality of the elements accessed. (2) strided: memory instructions use a base address and a parameter that refers to the increment in memory between elements which are adjacent in the vector register. (3) indexed: also known as gather/scatter, the instructions use a base address and an additional vector register of offsets.

Unit-stride and strided instructions calculate their addresses formulaically and the number of cycles spent performing the address generation depends on the number of cache lines needed to fulfil the request, e.g. four cache lines accessed would require four cycles in the functional unit. Indexed memory instructions require adding an offset to a base address and require $\frac{V L}{\text { lanes }}$ cycles to perform address generation where $V L$ is the vector length of the instruction. Using an existing technique [14], [35], [36], the vector register file bypasses the L1-d cache and goes directly to the L2 cache. This way more bandwidth can be provided at the expense of higher latency; it has been shown in the cited work that this extra latency is easily amortised due the large number of elements operated on per individual instruction. The number of cycles needed to complete a memory instruction depends on how many individual cache lines are requested and whether or not these are already resident in the L2 cache.

To operate on the vector registers, we have added a suite of non-memory vector instructions which is summarised in Table III. Each instruction requires $\frac{V L}{\text { lanes }}$ cycles to pass through a functional unit, with the exception of mask instructions which require one cycle. Comparison instructions produce a result to a mask register. Most instructions can be optionally masked with the exception of the permutative instructions, which rearrange the order of the input vector's elements. merge and iota are instructions found in the CRAY-1 [13].

TABLE III. NON-MEMORY VECTOR INSTRUCTIONS

\begin{tabular}{|l|l|}
\hline class & instructions \\
\hline initialisation & set all, clear all, iota \\
arithmetic & integer add, subtract \\
bitwise logical & and, shift left, shift right \\
comparison & greater than, less than \\
mask & set, clear, not, popcount \\
permutative & compress, shuffle, reverse \\
other & merge, copy, get/set element \\
\hline
\end{tabular}

\section{Evaluation OF Existing Sorting Algorithms}

This section presents an evaluation of three existing vectorised sorting algorithms: quicksort, bitonic mergesort and radix sort. The former two algorithms are classified as comparative sorts whereas the latter is a non-comparative sort. While comparison sorts generally have fewer limitations and facilitate simpler implementations, non-comparison sorts are still suitable in many scenarios at the expense of tailoring the algorithm to a specific datatype. Each algorithm chosen is suitable for a DLP-accelerated processor but they have very different characteristics from one another. Their strengths and weaknesses are found experimentally and used to guide our own algorithm in the next section.

Each algorithm is evaluated with three datasets, a MVL varying between eight and sixty-four elements and between one and four lockstepped parallel lanes. Based on these initial experiments we further evaluate some of the algorithms with additional configurations. All results use the metric cycles per tuple (CPT), that is the total execution time of the algorithm in cycles divided by the length of the input $n$.

The three principal datasets are small, medium and large which contain 51,200, 512,000 and 5,120,000 tuples respectively. There are several reasons why these datasets have been chosen. Firstly, small is able to reside in the L2 cache while the latter two increase in length by one and two orders of magnitude respectively. This helps identify performance issues related to the input size and also to pinpoint any noteworthy effects due to the cache. Secondly, these input lengths are multiples of the MVL however they are not perfect powers of two. Using perfect power of two lengths can often lead to outliers in trends. We have observed some strided memory patterns impacting performance negatively when $n$ is a power of two and for this reason we prefer to show the general case rather than the exception. 
Each dataset contains a random uniform distribution of 32-bit integer values. Although it is true that some sorting algorithms exhibit different behaviours when the input is not uniformly distributed, out of the algorithms presented in this work only quicksort would be affected. A random uniform distribution will at least present quicksort's average case behaviour.

Merely sorting an array of integers has very limited applications, we therefore create the datasets with both key and payload values; this opens up the applicability of these algorithms to very useful scenarios like database management systems. The two-value tuples are 64 bits each and have been organised as a structure of arrays which is common in applications like column-store DBMSs [37], [38].

A purely scalar algorithm called reference is also included with the results of each vectorised sorting algorithm, this allows for a common baseline when comparing the vectorised algorithms to one another. We use an in-place quicksort using a median of three pivot selection and an insertion sort cleanup; this variant of quicksort is known to perform well [39]. We have optimised all these algorithms by hand and have used software prefetching when beneficial.

\section{A. Quicksort}

Quicksort [39] is a comparison sort that uses a divide and conquer strategy to iteratively partition its input until it is sorted. It is well known for having fast implementations due its $O\left(n \cdot \log _{2} n\right)$ average complexity, cache-friendly memory access patterns and the simplicity of the algorithm's main body.

A vectorised version was first proposed for the CDC STAR [6]. Its implementation is recursive, stable and has relatively few operations per pass. It is not in-place and thus requires an auxiliary array to store partial results. The algorithm has a unitstride memory access pattern thus making it very bandwidth friendly. The original algorithm has been optimised further by using the median of three technique suggested in [39] for choosing the pivot. The pseudocode is shown in Fig.1; we have simplified it by omitting any vector stripmining code.

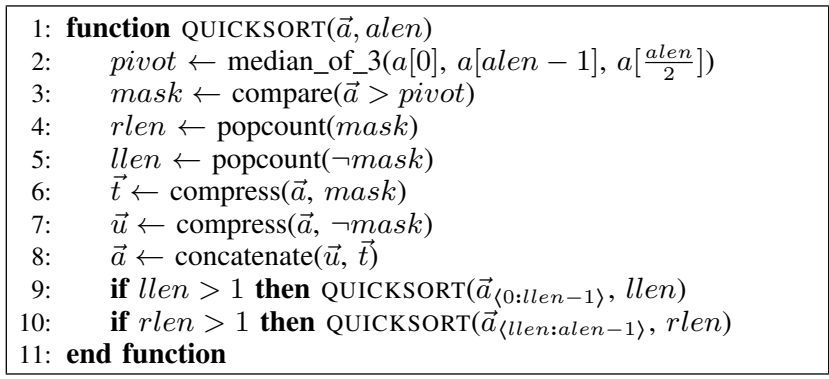

Fig. 1. Pseudocode for the vectorised quicksort algorithm

Fig.2 displays the results of this algorithm. The maximum speedup over the reference benchmark is $\mathbf{1 . 4 x}, \mathbf{1 . 6 x}$ and $\mathbf{1 . 8 x}$ for small, medium and large respectively. Going from a MVL of eight to sixteen increases the performance in all cases and is more pronounced than subsequent increases of the MVL. This can be explained by the fact that sixteen 32-bit elements accessed in a unit-stride fashion is exactly one cache line.
Increasing the number of lanes can help the algorithm's performance a little bit as the compare and compress instructions can benefit. Adding more than two lanes yields very little extra performance, at this point the memory operations dominate the execution time and none of these can be accelerated with lanes, i.e. there are no indexed memory operations.

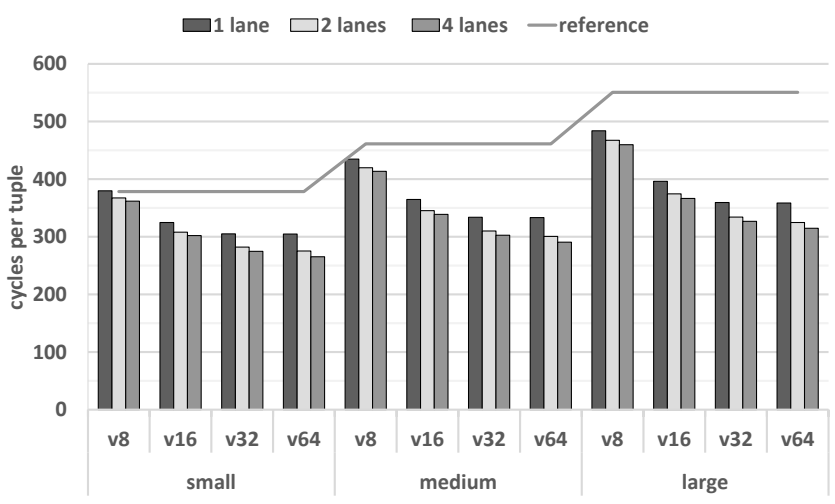

Fig. 2. Results for quicksort

Increasing the MVL from 32 to 64 only improves the performance marginally due to quicksort's divide and conquer strategy. As partitions get smaller, the effective vector length per function call is reduced and eventually causes serialisation which leads to an increased CPT. Table IV illustrates this for a MVL of 64 and the large dataset. The leftmost column shows the percentage of time spent processing partitions with a length in the range of from and to. freq displays the frequency of calls to the function operating on a partition with a length within the specified range. The highlighted column, avg cpt, indicates the average CPT measured in the main body. Going from top to bottom, it can be seen that the CPT remains similar until reaching partitions of 64 elements or fewer. At this point the vector registers become underutilised and serialisation begins. It can be seen that $62 \%$ of the total execution time is spent operating on partitions with fewer elements than the MVL.

TABLE IV. QUICKSORT'S PERFORMANCE PER PARTITION

\begin{tabular}{|r|rrr|r|}
\hline \% time & from & to & freq & avg cpt \\
\hline 15.6 & $5,120,000$ & 119,417 & 72 & 6.31 \\
10.6 & 119,416 & 2,786 & 3,091 & 4.43 \\
6.2 & 2,785 & 228 & 35,350 & 4.99 \\
5.3 & 227 & 65 & 94,389 & 8.56 \\
10.4 & 64 & 19 & 306,189 & 18.43 \\
19.8 & 18 & 6 & 849,856 & 41.11 \\
32.1 & 5 & 1 & $2,100,685$ & 92.15 \\
\hline
\end{tabular}

To help alleviate this problem, we use a variant of the algorithm. The work of [4] modifies the original algorithm and uses a vectorised odd-even transposition (OET) sort [40] as a cleanup mechanism. When the quicksort phase creates partitions equal or less to some predefined threshold, it gracefully returns instead of continuing recursively. When this modified quicksort finishes, OET sort is applied to the entire dataset. Typically this algorithm has a complexity of $O\left(n^{2}\right)$ but in this case it is only $O(n \cdot$ threshold $)$. We have found empirically a value of 16 to be optimal for the threshold.

Fig. 3 displays the results of the modified algorithm. The maximum speedup over the reference benchmark is $\mathbf{2 . 4 x}$, 
2.5x and 2.6x for small, medium and large respectively. On average these results are $\mathbf{1 . 4 x}$ better than the original quicksort implementation. A huge advantage of OET sort is that it operates on the entire dataset without concerning itself with partition boundaries and therefore leverages the entire MVL of the configuration. A disadvantage is that the cleanup algorithm suffers from a complexity of $O(n \cdot$ threshold $)$ and we have observed that increasing the threshold higher than 16 will cause the overall performance to decrease.

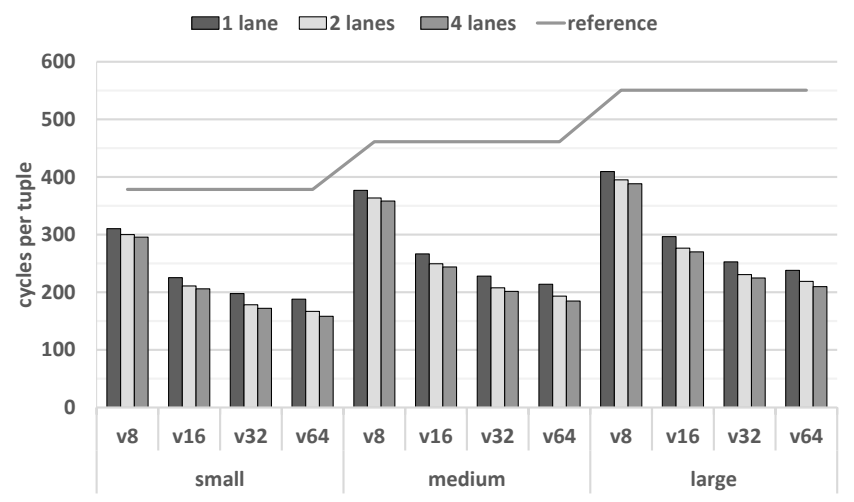

Fig. 3. Results for quicksort with OET cleanup

\section{B. Bitonic Mergesort}

This subsection explores bitonic mergesort, a type of algorithm that makes use of sorting networks [41]. Sorting networks differ from many other categories of sorting algorithms in that their complexity and number of operations are fixed ahead of time and do not depend on the values of the input. The algorithm is broken into two phases. Phase $\mathbf{1}$ creates relatively short sorted blocks from the input. Phase $\mathbf{2}$ iteratively merges these blocks into a single sorted block.

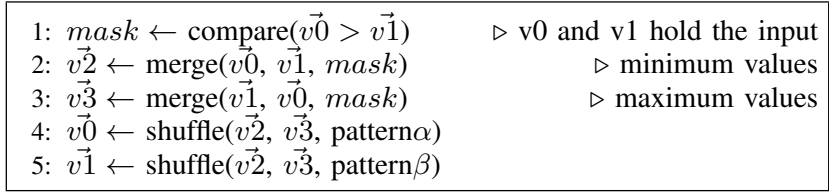

Fig. 4. Pseudocode for a single step of the vectorised bitonic network

Phase 1 loads a block of $2 \cdot M V L$ contiguous elements into two vector registers, sorts them using a bitonic sorting network and stores the sorted output to memory. Sorting $x=2 \cdot M V L$ elements requires $\sum_{i=1}^{\log _{2} x} i$ steps. The pseudocode of a single step is shown in Fig. 4 and has five non-memory vector instructions (nine when using a payload). Each step uses two unique shuffle patterns which are pregenerated before compilation. Increasing the MVL also increases the number of steps needed, however, all of the instructions within a step can be accelerated using parallel lanes. This phase creates $\frac{n}{2 \cdot M V L}$ sorted blocks which are used in the next phase. For more information regarding vectorised bitonic sorting networks we refer the reader to [2], [6].

Phase 2 iteratively merges adjacent sorted blocks into larger sorted blocks until finally producing a single sorted block. At this point the blocks produced from Phase 1 are already larger than the MVL meaning that they must be merged piece by piece rather than all at once. To accomplish this we use a technique proposed in [3]. Merging two blocks of combined length $m$ requires $\left\lfloor\frac{m-1}{M V L}\right\rfloor$ calls to a bitonic merging network. Bitonic merging networks are similar to the sorting networks described in Phase 1 , however as both inputs are already sorted they need only $\log _{2} x$ steps instead of $\sum_{i=1}^{\log _{2} x} i$ steps. This merging strategy has a linearithmic complexity of $O\left(n \cdot \log _{2}\right.$ blocks $)$ where blocks is the number of sorted blocks created in Phase 1. For more information about iterative block merging using bitonic merging networks we refer the reader to [1], [3], [5].

Fig.5 displays the results of bitonic mergesort. It can be seen that for all the single lane experiments, increasing the MVL degrades the CPT instead of improving it. This is because, as previously mentioned, increasing the MVL increases the number of steps in the sorting network and therefore adds extra work. Using multiple lanes allows this work to be done in parallel. It can be seen that four lanes is enough to overcome the penalty of increasing the MVL and using two lanes has mixed results due to the two opposing effects. The maximum speedup of bitonic mergesort over the reference benchmark is 2.9x, 3.0x and 2.9x for small, medium and large respectively.

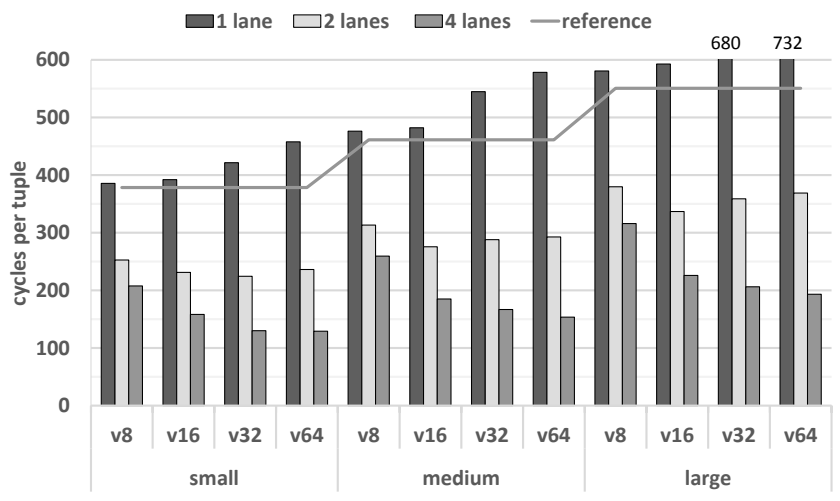

Fig. 5. Results for bitonic mergesort

Fig.6 shows the trend for each dataset run with a MVL of 64 and varying the number of lanes from one to 64 . When 64 lanes are used the maximum speedup over the reference benchmark is $6.4 x, 6.9 x$ and $6.4 x$ for small, medium and large respectively. Increasing the number of lanes beyond 16 yields very little extra benefit. This shows that while a large number of lanes is useful in this case, increasing this number all the way to the MVL has disproportionate returns. One drawback of this approach is that it requires a general shuffle vector instruction. As the MVL of the vector registers increases, this instruction becomes more complex to implement with a low number of cycles. Intel's AVX2 offers an eight-element 32-bit general shuffle instruction with a 3-cycle latency. The Intel Xeon Phi has a sixteen-element 32-bit general shuffle and an average latency of 6 cycles has been measured [42]. This would mean their shuffle instructions require 0.375 cycles per element. All non-memory vector instructions run in our simulation environment assume a latency of $\frac{V L}{\text { lanes }}$ therefore the four-lane configurations require a lower 0.25 cycles per element. This implies that our configurations with more than four lanes assume an aggressive implementation of shuffles 
and the results should be considered optimistic.

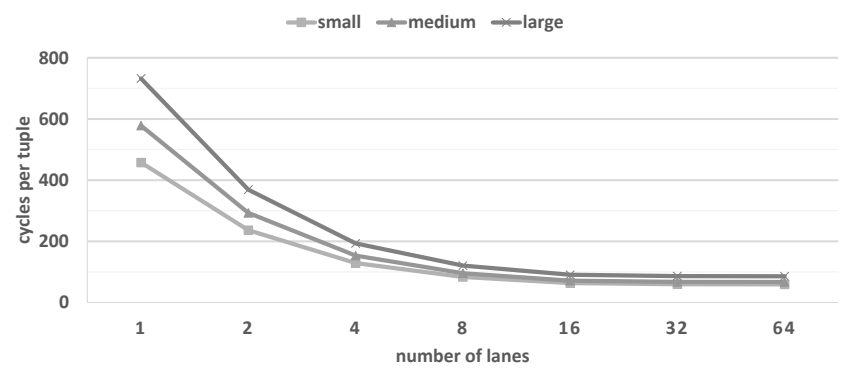

Fig. 6. Bitonic mergesort: varying lanes when $M V L=64$

\section{Radix Sort}

Radix sort [43] is a non-comparative numerical sort with a complexity of $O(k \cdot n)$ where the value $k$ depends on the number of passes of the algorithm. For m-bit integers, the number of passes is $\left\lceil\frac{m}{\log _{2} \text { bins }}\right\rceil$ where bins, an input parameter of the algorithm, refers to the size of the histogram used internally and $\log _{2}$ bins is the number of bits sorted per pass.

The work of [7] proposed a technique to vectorise the algorithm that was originally implemented on the CRAY YMP. Fig.7 provides a step by step example in order to give a high-level overview of the algorithm. The three steps together make up the body of the main loop that forms a single pass of the algorithm. For a more detailed explanation of the vectorised radix sort algorithm we refer the reader to [7].

Step 1 The entire input is loaded iteratively and a histogram is created for a subset of the input's bits, for example, the first pass of the algorithm uses the first $\log _{2}$ bins bits of each value, the second pass uses the next $\log _{2}$ bins bits, etc. Subfigures $7 \mathrm{i}$ and 7ii illustrate a single iteration of the vectorised loop implementing this step; in total there are $\frac{n}{M V L}$ iterations. (a) The input array is loaded into a vector register and $\log _{2}$ bins bits of these values are extracted. Due to the nature of the algorithm, each vector element must see a contiguous portion of the dataset therefore the input is loaded using strides of $\frac{n}{M V L}$ and the base address is incremented after every iteration. (b) The extracted bits are indices into a histogram which is incremented. Multiple elements within the same vector can index to the same bin. If a single histogram were used, updates using these indices would be incorrect. To avoid such conflicts, a local histogram is used for each vector element, i.e. there are $M V L$ local histograms. Step 2 A prefix sum is performed over the collection of local histograms. Step $\mathbf{3}$ The entire input is loaded again and distributed to an output array with offsets determined by the prefix sum. Similar to Step 1, Subfigures $7 \mathrm{iv}, 7 \mathrm{v}$ and $7 \mathrm{vi}$ illustrate a single iteration of the vectorised loop. (a) The input is reloaded into a vector register in an identical way to Step 1a and the same bits are extracted. (b) The extracted bits are indices into the prefix sum which is read and incremented. Unlike Step 1b, the values before the increment are retained. (c) The retained values are used as a vector of offsets to scatter the input values to an output array. This output array becomes the input array in the next pass of the algorithm. The algorithm guarantees that all elements are scattered to unique locations.
We first look for the optimal number of bins for a given dataset. Fig.8 shows the average CPT for each of the datasets as the number of bins is increased from four to 1,024. Each experiment is run with a MVL of 64 and a single lane. As the number of bins is increased the number of passes is decreased, e.g. four bins require sixteen passes and 256 bins require four passes. Increasing the number of bins also increases the memory needed and reduces the locality of the working set, this has the effect of increasing the number of cycles needed per pass. For all the datasets we find the sweet spot to be sixteen bins.

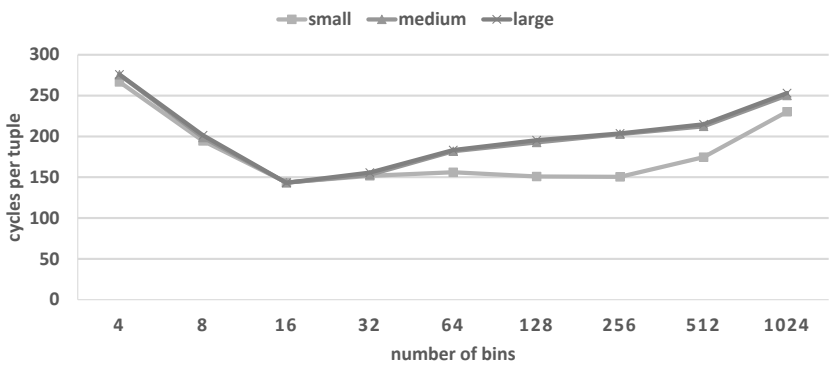

Fig. 8. Radix sort: optimal number of bins when $M V L=64$

Fig.9 shows the results of radix sort executed with sixteen bins. The values follow a nice trend in that increasing the MVL decreases the average CPT. The lanes also help, however similar to quicksort, adding more than four would give disproportionate returns. Although the indexed memory instructions can benefit from using lanes, the algorithm is still dominated by its strided memory access pattern which does not benefit from using them. Another interesting property is that each dataset exhibits very similar behaviour in terms of CPT; this is because radix sort has a complexity of $O(k \cdot n)$. The maximum speedup over the reference benchmark is $\mathbf{3 . 6} \mathbf{x}$, 4.3x and 5.2x for small, medium and large respectively.

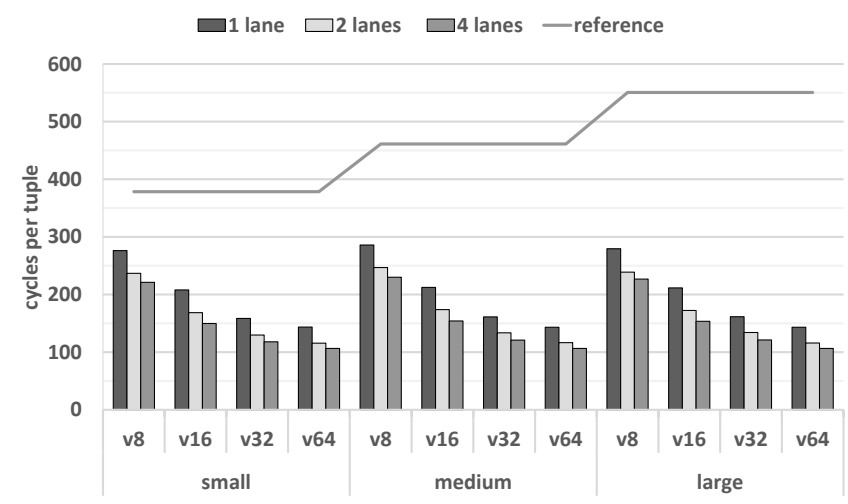

Fig. 9. Results for radix sort when bins $=16$

There are two significant drawbacks to this algorithm. (1) Each pass of the algorithm must be stable, i.e. equal values do not change their relative order. To ensure stability, a technique called loop raking is used which partitions the input into $M V L$ chunks of sequential values; each element of the vector register, termed 'virtual processor', operates on its own chunk. This requires using a strided memory access pattern which guarantees that any element of the vector register 


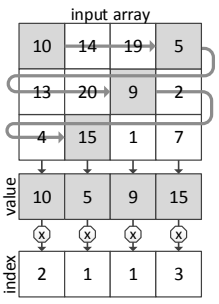

(i) Step 1a

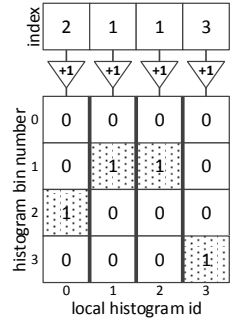

(ii) Step $1 b$

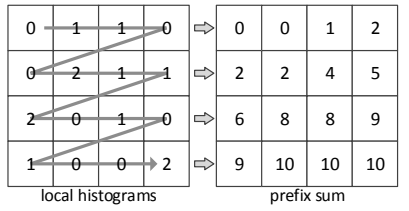

(iii) Step 2

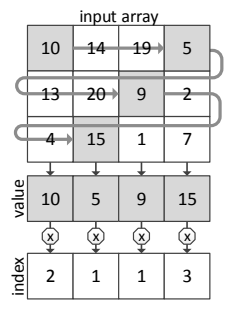

(iv) Step 3a

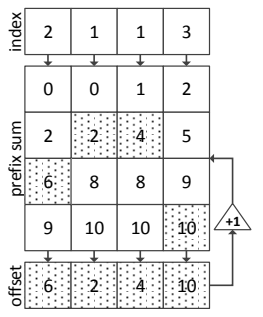

(v) Step 3b

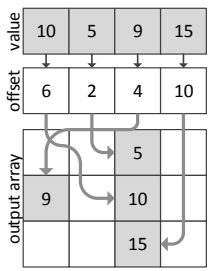

(vi) Step 3c

Fig. 7. The three steps of the first pass of the vectorised radix sort where $n=12, M V L=4$ and bins $=4$

sees a contiguous portion of the input. As the input length $n$ increases, so does the stride; this consequently reduces spatial locality and underutilises the available memory bandwidth. (2) In order to avoid conflicts, the algorithm's histogram must be replicated $M V L$ times. This limits the feasible number of bins which the algorithm can use without encountering cache locality problems and in turn directly influences the number of passes the algorithm must make. This replication also increases the work necessary to calculate the prefix sum in Step 2.

\section{Summary}

We have evaluated three distinct sorting algorithms on a uniform platform using consistent metrics. Although all these algorithms outperformed the scalar reference, each one suffered from unique weaknesses and bottlenecks.

Quicksort was shown to perform very well when working with partitions much larger than the MVL, however as the size of the partition decreased the performance of quicksort degraded. We were able to rectify this somewhat by adopting a variant of the algorithm that uses OET sort as a cleanup mechanism. Increasing the parametrisable threshold reduced the amount of time that quicksort spent processing short partitions. However, due to the fact that the OET sort has an $O\left(n^{2}\right)$ complexity, we observed that we could not increase the threshold indefinitely without degrading the overall performance.

Bitonic mergesort was shown to perform better, but only when the number of parallel lanes is high. Four parallel lanes were needed to outperform quicksort and even more were needed to outperform radix sort in most cases. When a single-lane configuration was used, the algorithm performance degraded as the MVL increased. This behaviour is in stark contrast to that of quicksort whereby the algorithm performed better as the MVL grows but exhibited only marginal speedups when adding parallel lanes. Additionally, bitonic mergesort requires a general shuffle instruction which is complicated to implement with a low latency when the MVL is large.

Both quicksort and bitonic mergesort suffer from a complexity of $O\left(n \cdot \log _{2} n\right)$ whereas radix sort has a highly desirable complexity of $O(k \cdot n)$. In contrast to bitonic mergesort, radix sort works well on SIMD hardware with or without parallel lanes. It also does not get bottlenecked by a divide and conquer strategy causing low effective vector lengths as is the case with quicksort. Unfortunately, radix sort suffers from using a stride in its dominant memory access pattern as well as having to replicate its internal bookkeeping structures by the number of elements in a vector register. This is in contrast to other algorithms which primarily use unit-stride access patterns and have very modest memory requirements. We use these weaknesses to motivate the next section of our work.

\section{VSR SORT}

This section presents Vectorised Serial Radix (VSR) sort, our novel vectorised non-comparative sorting algorithm based on radix sort. We first explain and discuss the algorithm. After, we introduce two new vector instructions in order to facilitate the algorithm's vectorisation; we also propose a corresponding hardware structure to implement these instructions. Finally, we evaluate the algorithm experimentally and compare the results and behaviour with those of the previous algorithms.

\section{A. The Algorithm}

We developed VSR sort with the aim to be efficiently vectorisable without the drawbacks identified in Section III. VSR sort is inspired by radix sort and follows more closely a serial implementation of the algorithm. Instead of accessing the input with a large strided access pattern, a much more efficient unitstride access is used. Additionally, the bookkeeping structure is not replicated $M V L$ times making the algorithm a lot more cache friendly. Fig.10 provides a high-level overview of one pass of the algorithm. Each step is analogous to its counterpart in Section III-C.

Step 1 The input is loaded iteratively and a histogram is created for a subset of the input's bits. (a) The input is loaded into a vector register and $\log _{2}$ bins bits of these values are extracted. Unlike the vectorised radix sort, a unit-stride access pattern is now used. (b) The extracted bits are indices into a histogram which is incremented. Instead of $M V L$ local histograms, only a single instance is used. Conflicting indices are detected dynamically and the increment value is modified accordingly. This conflict detection/correction is discussed in the next subsection. Step 2 A prefix sum is performed over the histogram. Fewer operations are needed than the previous implementation due to the single histogram. Step 3 The entire input is loaded again and distributed to an output array with offsets determined by the prefix sum. (a) The input is reloaded into a vector register in an identical way to Step 1a and the same bits are extracted. (b) The extracted bits are indices into the prefix sum which is read and incremented. The prefix sum is updated, however, unlike the previous implementation the increment may be more than 1 due to conflicting indices. The values loaded from the prefix sum are corrected according to the conflicts; these corrected values become the offsets used in 
of each instruction in $O\left(M V L^{2}\right)$ time however this would not be very useful in this context for several reasons. (1) These instructions occur on the critical path of tight loops executed many times and such suboptimal implementations would negatively impact performance due to very long pipeline stalls. (2) The goal is to propose a vectorised sorting algorithm that scales well with the MVL; an implementation of $O\left(M V L^{2}\right)$ complexity becomes less practical each time the MVL is enlarged. (3) In VSR sort, both instructions do similar work, use the same input and are always scheduled back to back; calculating both results separately would be doing redundant work. The implementation we propose can calculate VPI and VLU together in $2 \cdot M V L$ cycles. After, we propose a variant that can reduce the execution time to approximately $\frac{2 \cdot M V L}{\text { lanes }}$ cycles.

To achieve this, a content-addressable memory (CAM) with $M V L$ entries is used. Each CAM entry contains a key entry and a valid bit used for the lookup, and two possible return values: count and last idx. count is the running total of the number of times a particular value of the input vector register has been seen. last idx is an index of the input vector register and refers to the last position where a particular value has been observed. Calculating an element of the output vector register requires two cycles; one for reading the CAM and another for updating it.

Fig.14 shows an example of the process to calculate VPI. The diagram shows the state of the hardware before the instruction has completed. Six of the eight elements have already been calculated (shown with a dotted pattern) and the seventh element is just about to be calculated. Arrows with a solid line represent activity done on the first cycle and arrows with a dashed line represent activity done on the second. The value 9 located at index 6 of the input is used to access the CAM. The value located in the count field is copied into the seventh element of the output vector register. This value is 1 because there has been exactly one element of input seen up until this point in time which contains the value 9. On the second cycle count is incremented and the corresponding last $i d x$ value will be updated with 6 as this refers to the most recent index of the input vector register where the value 9 was observed.

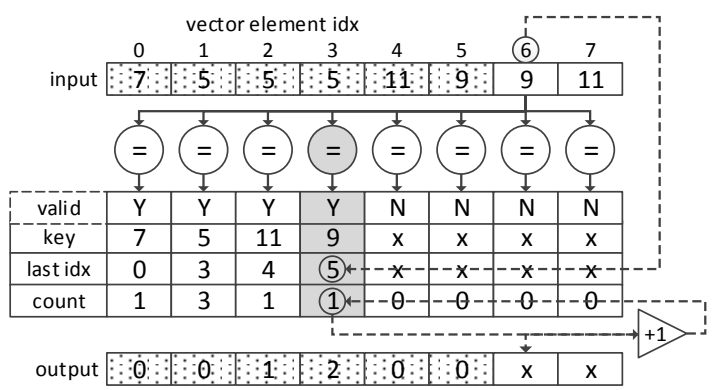

Fig. 14. Proposed hardware calculating VPI

last idx is not used to calculate VPI however it is relatively simple to update this field when updating count, this way when VLU is executed after VPI using the same input, all that remains to be done is to convert the array of last idx values to a bitmask. This can be done in relatively few cycles.
Although the CAM requires $M V L$ entries to cover a worsecase scenario, the size of each field can be relatively small. valid is a single bit and both count and last idx require $\log _{2} M V L$ bits. To keep the implementation general purpose, we make key 32 bits.

One obvious obstacle extending this implementation to multiple lanes is that the semantics of the instructions VPI and VLU are defined serially. In a similar vein to ILP in OoO superscalar microprocessors, we propose finding the DLP dynamically during runtime. Adjacent elements of the input vector register are arranged into groups; the elements within a group can be processed in parallel provided they do not conflict with one another otherwise they are processed serially. Detecting conflicts requires $\frac{l !}{2 \cdot(l-2) !}$ comparators where $l$ is the number of parallel lanes targeted, i.e. the group size. To keep the number of comparators low, a small $l$ is expected to be chosen. We distinguish the conventional parallel lanes used in a vector unit from the group size of VPI/VLU which we will refer to as CAM lanes.

Fig.15 illustrates the parallel optimisation using two CAM lanes. There is an input vector register which is processed from left to right and underneath there are two timelines that represent the relative execution time of both the parallel and serial implementations. Each block of the timelines represents two execution cycles however the parallel timeline shows stacked blocks meaning these are processed in tandem. The first, third and fourth groups of elements can be processed in parallel as there are no conflicts. The second group of elements has a conflict and is serialised. The hatched box represents the time saved over the serial implementation. In this case it is $\frac{3}{8}$ blocks or a 1.6x speedup.

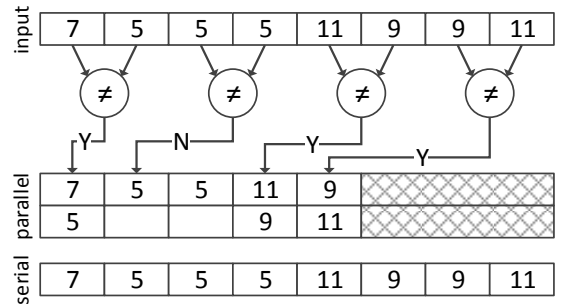

Fig. 15. Parallel optimisation for hardware with two CAM lanes

The benefit of a parallel implementation depends highly on the input. For VSR sort it is the number of histogram bins that will determine the probability of a conflict within a group. Increasing the number of bins decreases the number of collisions and thus allows for more parallelism. This is a tradeoff because increasing the number of bins also increases the cache footprint of the algorithm. The probability of not having a conflict for $b$ bins and $l$ CAM lanes is $\frac{b !}{b^{l} \cdot(b-l) !}$ when $l \leq b$. Table $\mathrm{V}$ shows the probability of group collisions calculated from the formula with various combinations of bins and CAM lanes. As $l$ increases the probability of a collision approaches 1.0 and all blocks are serialised with a net result performance equal to the implementation with a single CAM lane.

We propose choosing two or four CAM lanes as increasing the parallelism further would give diminishing returns due to a high amount of serialisation. Increasing the CAM lanes 
TABLE V. Probability of COLLISIONS USING CAM LANES

\begin{tabular}{|r|c|c|c|c|}
\hline bins & \multicolumn{4}{|c|}{ CAM lanes } \\
\hline & 2 & 4 & 8 & 16 \\
\cline { 2 - 5 } 4 & 0.25 & 0.91 & 1.00 & 1.00 \\
16 & 0.06 & 0.33 & 0.88 & 1.00 \\
64 & 0.02 & 0.09 & 0.37 & 0.87 \\
256 & 0.00 & 0.02 & 0.10 & 0.38 \\
1,024 & 0.00 & 0.01 & 0.03 & 0.11 \\
\hline
\end{tabular}

further would also require (a) increasing the complexity of the CAM with more ports and (b) increasing the number of comparators used to detect collisions. A configuration with four CAM lanes requires a modest six comparators whereas a configuration with eight CAM lanes would require 28 comparators. Detecting conflicts in this way resembles the logic needed to detect hazards in superscalar register renaming. In subsequent experiments, we set the number of CAM lanes equal to the number of conventional vector lanes and refer to both together as lock-stepped lanes, or simply as lanes.

In order to verify our timing assumptions, we model this structure using CACTI-P [47]. We choose a $32 \mathrm{~nm}$ technology and a $V_{d d}$ of $0.75 \mathrm{~V}$ which matches our baseline Westmere microarchitecture. Assuming a CAM of 64 entries and between one and four ports, our experiments show that searching and accessing this structure together requires between $0.276 \mathrm{~ns}$ and $0.339 \mathrm{~ns}$. These values are lower than the $0.375 \mathrm{~ns}$ cycle time of our simulated processor which indicates it is reasonable to assume updating the structure can be done in two cycles per element. In Section IV-C we make further experiments assuming different latencies to measure the impact of alternative hardware implementations.

\section{Results}

To directly compare VSR sort with the previous vectorised radix sort, we first run it with sixteen bins as this was the optimal value found in Section III-C. Fig.16 displays the results using single-lane configurations. On average VSR sort performs 1.8x faster than the previous algorithm; in this case the constant factor of $O(k \cdot n)$ has been improved.

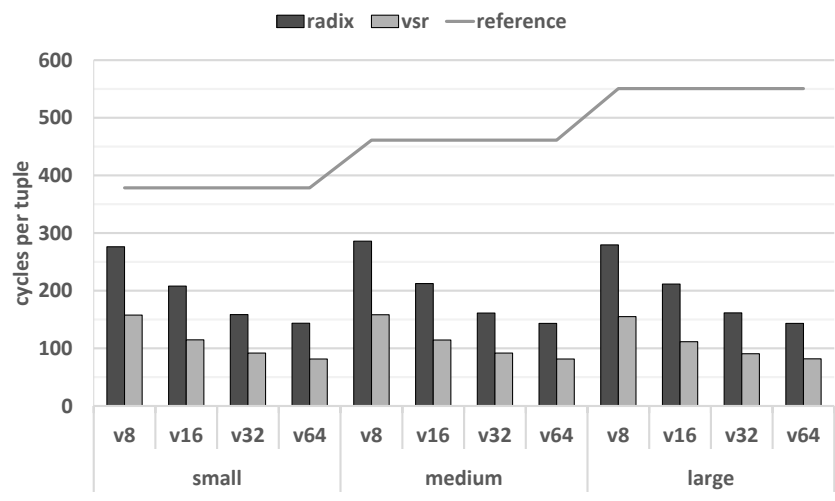

Fig. 16. Radix and VSR sort when lanes $=1$ and bins $=16$

Because we have eliminated the histogram replication of the previous vectorised radix sort, it should be possible to increase the number of bins without compromising performance and thus reduce the number of passes. Fig. 17 shows VSR sort run with a MVL of 64 and a single lane while varying the number of bins. In comparison to the equivalent experiment in Section III-C, the optimal number of bins for VSR sort is 256 (four passes) instead of sixteen (eight passes).

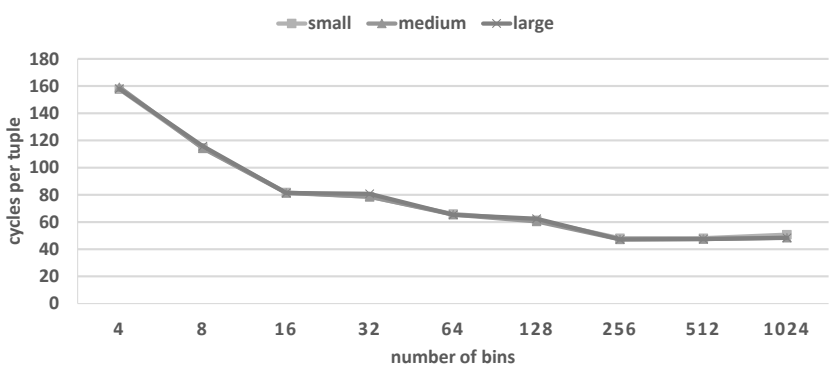

Fig. 17. VSR sort: optimal number of bins when $M V L=64$

Fig. 18 shows the results of VSR sort run with 256 bins. On average it performs $\mathbf{1 . 8 x}$ faster than VSR sort run with sixteen bins; in this case the $k$ from $O(k \cdot n)$ has been improved. With a single-lane configuration the maximum speedup over the reference is $\mathbf{7 . 9 x}, \mathbf{9 . 8 x}$ and $\mathbf{1 1 . 7} \mathrm{x}$ for small, medium and large respectively. In our experiments, this is the highest speedup seen so far including other algorithms run with parallel lanes. When this algorithm is run with parallel lanes the maximum speedup over the reference benchmark is $14.9 x, 17.3 x$ and 20.6x. On average, VSR sort performs $\mathbf{3 . 4 x}$ better than the next-best vectorised sorting algorithm when run on the same hardware configuration.

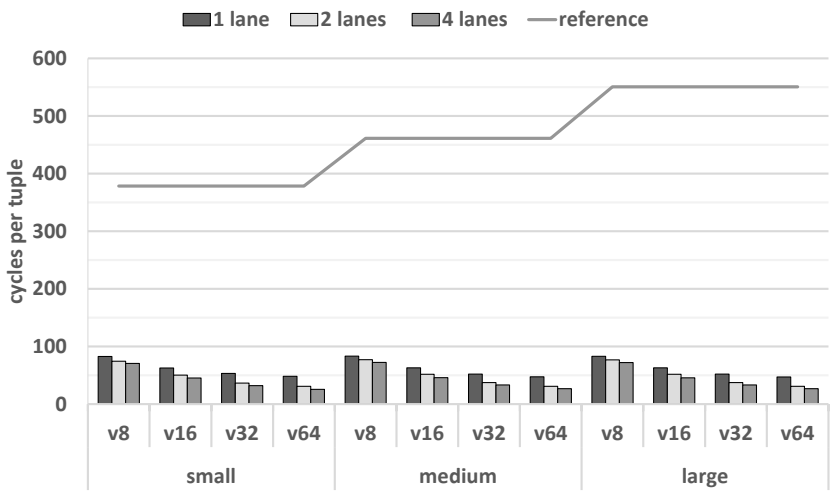

Fig. 18. Results for VSR sort when bins $=256$

There are some noteworthy observations about VSR sort. Firstly, while the algorithm benefits from longer MVLs, very good results can still be achieved with a short MVL. Using a MVL of eight can achieve a CPT below 100 for all datasets whereas the algorithms evaluated in Section III could not achieve this kind of performance even with a MVL as large as 64. Only bitonic mergesort run with at least sixteen parallel lanes could achieve an average CPT lower than 100 for all the datasets. Secondly, while lanes give a clear advantage, the performance of VSR sort when run with a single lane is commendable. VSR sort can achieve an average CPT below 50 on the single-lane configurations; even bitonic mergesort run with a large number of lanes could not achieve a CPT below 60 . Thirdly, it can be seen that all the datasets achieve similar CPTs to each other. This implies that the algorithm's 


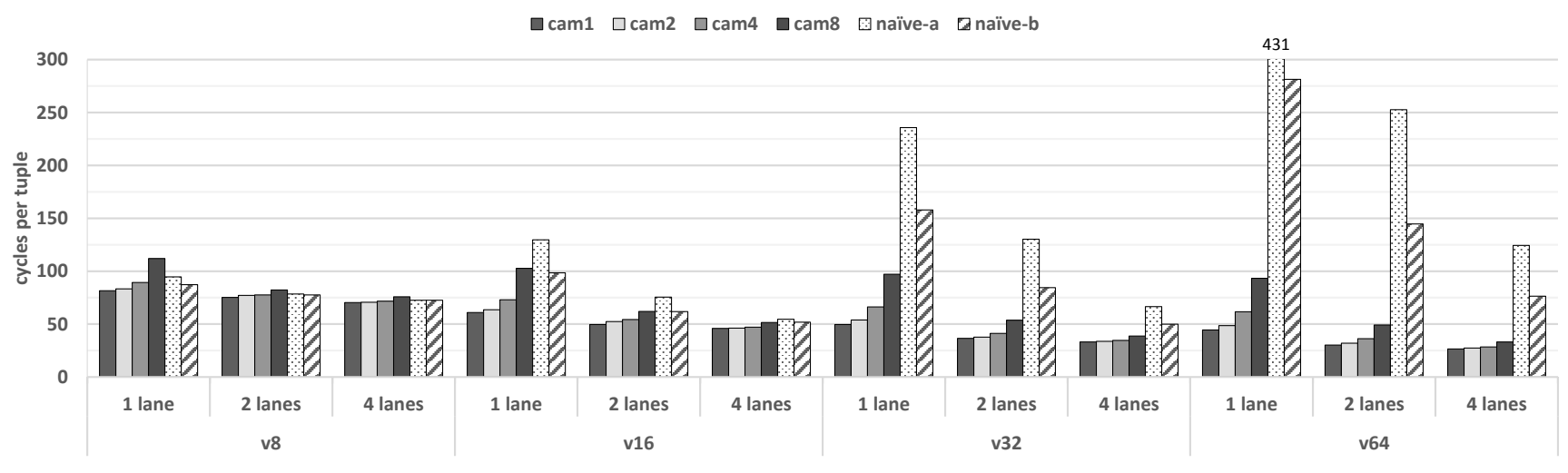

Fig. 19. Results for VSR sort when processing the large dataset run with various hardware implementations of VPI and VLU

complexity of $O(k \cdot n)$ is being adhered to. The original vectorised radix sort also has a complexity of $O(k \cdot n)$ however our VSR sort improves $k$ as well as the runtime constant.

We conduct additional experiments with alternative implementations of VPI and VLU. Fig.19 shows the results of VSR sort executed with the large dataset run with six alternative hardware configurations. cam1, cam2, cam4 and cam8 fix the CAM's latency to one, two, four and eight cycles per element respectively; cam 2 being the default configuration used in the previous experiments. naïve- $a$ removes the CAM structure and calculates VPI and VLU separately using a simple hardware implementation with quadratic complexity. Each instruction requires $\sum_{i=1}^{V L-1}\left\lceil\frac{i}{\text { lanes }}\right\rceil$ cycles where $V L$ is the vector length of the operation. naïve- $b$ has a similar implementation to naïve$a$ however both VPI and VLU are calculated simultaneously and the latency is paid just once.

In all cases, increasing the latency of the CAM structure decreases the overall performance of VSR sort. What is notable is that the impact on performance is far more pronounced when only one lane is used; as the number of lanes is increased, the relative penalty of a higher latency CAM decreases. For example, using a MVL of 64 and one lane, the increase in CPT when changing from cam 2 to cam 4 is $27 \%$. In contrast, when four lanes are used the increase in CPT is only $3 \%$. Likewise, when moving from $\operatorname{cam} 2$ to $\operatorname{cam} 8$, the single-lane configuration has a $92 \%$ increase in CPT whereas the fourlane configuration's increase in CPT is a milder $21 \%$. Using the more aggresive caml configurations yields speedups over our default cam 2 configuration between 1.01x and 1.09x. In contrast, using the less aggressive cam 4 configurations over cam2 exhibits CPT increases between $1 \%$ and $27 \%$. Our default latency of two cycles per element appears to be a good compromise between cam1 and cam4, however, the results also suggest that it is possible to use less aggressive CAM-based implementations of VPI/VLU and still have good performance.

Both nä̈ve- $a$ and nä̈ve- $b$ have different trends to the CAMbased implementations. Although using multiple lanes tempers the impact of higher latencies, we observe that increasing the MVL decreases the overall performance of the algorithm which is in contrast to the CAM-based experiments. It can be seen that for a MVL of eight, naïve- $a$ performs comparably with the CAM-based implementations and even outperforms cam8. Once the MVL is increased, however, the burden of the quadratic complexity takes effect. Even when multiple lanes are leveraged, they are not numerous enough to always counteract the effects of the $O\left(M V L^{2}\right)$ implementation. naïve$b$ can be seen as an optimisation of naive- $a$. When multiple lanes are used, naïve- $b$ can sometimes reach comparable performance to cam8, however in the case of $M V L=64$, even using four lanes naïve- $b$ has a $130 \%$ increase in CPT over cam8. We conclude that a CAM-based approach has clear performance advantages over either of the naïve approaches.

\section{RELATED WORK}

There have been numerous works related to sorting on SIMD architectures and the most relevant articles have already been cited and discussed in previous sections. There have also been several works that focus on the problem of using SIMDlike instructions to update data structures where there may be memory conflicts. We summarise these works and explain why they are different to our own instructions and why none of them are appropriate for VSR sort.

The work of [48] proposes scatterAdd, an instruction for stream architectures that provides support for parallel histogram generation. This instruction takes the following form: scatterAdd (base, $\overrightarrow{\text { ins }_{3}} c$ ) where base is a location in memory of a histogram, $\overrightarrow{\text { bins }}$ is an array of values that correspond to bins of the histogram and $c$ is the increment (typically 1). scatterAdd is advantageous in the sense that updating a histogram can be done with a single stream instruction whereas our approach requires a combination of six vector instructions to do the same. Our proposed instructions VPI and VLU can be used to emulate the behaviour of scatterAdd however the semantics are much stricter meaning they can also be used for VSR sort whereas scatterAdd cannot. scatterAdd is a FetchAndOp-style instruction but lacks a return path for the original values; furthermore, the instruction lacks deterministic ordering semantics. VSR sort requires a return path as well as strict serial ordering therefore scatterAdd is not suitable.

The work of [49] proposes atomic SIMD memory instructions. Gather/scatter functionality is added to a SSE-like ISA then this functionality is extended with load-linked/storeconditional semantics. The instructions use a best-effort implementation meaning that stores to conflicting addresses will fail and this is explicitly visible to the user in the form of a bitmask. When working with a data structure like a histogram, 
a SIMD gather-modify-scatter operation must be contained in a data-dependent loop and repeated until every element has been successfully updated. In the worst case this will have the penalty of complete serialisation including the overhead of the loop. Unlike scatterAdd, this technique has a return path meaning that it is possible to retrieve a copy of the original value before it is updated. Unlike VPI and VLU, there is no intra-register ordering guarantees which implies that this will not work for VSR sort in which the ordering is of utmost importance. The atomic SIMD memory operations also work between threads meaning that multiple cores with SIMD support can attempt to update the same data structure. VPI and VLU do not need such complexity because all input and output is contained within registers rather than shared memory.

Intel has recently announced AVX-512CD conflict detection instructions [10], an extension to AVX-512 with an initial implementation expected in 2015. VPCONF LICT uses a single multimedia register as input and returns a bitmask showing conflicting pairs. By using this bitmask it is possible to remedy gather-modify-scatter operations with conflicting indices and this could be used to aid histogram generation. Any indices that conflict can be masked out and similar to [49] the programmer must use a loop construct to iteratively process the same input until there are no pending elements. While we also generate histograms in VSR sort, this occurs in very tight loops on the critical path with a high number of iterations; a best-effort implementation could severely impact performance. We have proposed suitable instructions to aid this as well as implementations that are efficient and scalable. We avoid data-dependent loops to handle conflicts and we prevent unnecessary repeated accesses to the same position of the histogram structure. As of this moment both the implementation and performance characteristics of AVX-512CD are not public, it is therefore impossible to compare it with our proposal.

\section{CONCLUSIONS}

SIMD extensions have become ubiquitous in modern microprocessors and are expected to grow in width and functionality in future generations. In this article we have performed extensive analysis on three diverse sorting algorithms in the context of future SIMD support. We have learned that all of the algorithms suffer from bottlenecks and scalability problems due to the irregularity of the DLP and the limitations of a standard SIMD instruction set. Based on these findings we have proposed VSR sort, a novel way to efficiently vectorise radix sort. To enable this algorithm in a SIMD architecture we have defined two new instructions: vector prior instances and vector last unique. We have provided a suitable hardware proposal that includes both serial and parallel variants. We have demonstrated that the algorithm scales well when increasing the maximum vector length, and works well both with and without parallel lockstepped lanes. VSR sort has shown maximum speedups over a scalar baseline between 7.9x and $11.7 \mathbf{x}$ when a simple single-lane pipelined vector approach is used and maximum speedups between 14.9x and 20.6x when as few as four parallel lanes are used.

We have compared VSR sort with three very different vectorised sorting algorithms: quicksort, bitonic mergesort and a previously proposed implementation of radix sort. VSR sort outperforms all of the aforementioned algorithms and achieves a comparatively low CPT without strictly requiring parallel lanes. It has a complexity of $O(k \cdot n)$ meaning that this CPT will remain constant as the input size increases, a highly-desirable property of a sorting algorithm. The $k$ factor is significantly improved over the original vectorised radix sort as well as the constant performance factor. Its dominant memory access pattern is unit-stride which helps maximise the utilisation of the available memory bandwidth. Unlike the previous vectorised radix sort, VSR sort does not replicate its internal bookkeeping structures which consequently allows them to be larger and reduces the number of necessary passes of the algorithm. On average VSR sort performs 3.4x better than the next-best vectorised sorting algorithm when run on the same hardware configuration.

\section{ACKNOWLEDGMENT}

The authors would like to thank Morteza Biglari-Abhari for reading this work. We are largely indebted to the efforts of Michael Swift who meticulously reviewed and critiqued the text multiple times leading to a considerably improved publication. We would also like to thank all of our anonymous reviewers, especially reviewer B for his or her highly detailed and insightful comments which helped develop the article. The research leading to these results has received funding from the European Union's Seventh Framework Programme (FP7/20072013) under the AXLE project (GA $n^{\circ}$ 318633) and from the RoMoL ERC Advanced Grant (GA n ${ }^{\circ}$ 321253). Timothy Hayes is also supported by a FPU research grant from the Spanish MECD.

\section{REFERENCES}

[1] J. Chhugani, A. D. Nguyen, V. W. Lee, W. Macy, M. Hagog, Y.-K Chen, A. Baransi, S. Kumar, and P. Dubey, "Efficient Implementation of Sorting on Multi-Core SIMD CPU Architecture," Proceedings of the VLDB Endowment, vol. 1, no. 2, pp. 1313-1324, Aug. 2008.

[2] B. Gedik, R. R. Bordawekar, and P. S. Yu, "CellSort: High Performance Sorting on the Cell Processor," in Proceedings of the 33rd International Conference on Very Large Data Bases, ser. VLDB, 2007, pp. 12861297.

[3] H. Inoue, T. Moriyama, H. Komatsu, and T. Nakatani, "AA-Sort: A New Parallel Sorting Algorithm for Multi-Core SIMD Processors," in Proceedings of the 16th International Conference on Parallel Architecture and Compilation Techniques, ser. PACT, 2007, pp. 189-198.

[4] S. A. Levin, “A fully vectorized quicksort,” Parallel computing, vol. 16, no. 2, pp. 369-373, 1990

[5] N. Satish, C. Kim, J. Chhugani, A. D. Nguyen, V. W. Lee, D. Kim, and P. Dubey, "Fast Sort on CPUs and GPUs: A Case for Bandwidth Oblivious SIMD Sort," in Proceedings of the 2010 ACM SIGMOD International Conference on Management of Data, ser. SIGMOD, 2010, pp. 351-362.

[6] H. S. Stone, "Sorting on STAR," IEEE Transactions on Software Engineering, vol. 4, no. 2, pp. 138-146, Mar. 1978.

[7] M. Zagha and G. E. Blelloch, "Radix Sort for Vector Multiprocessors," in Proceedings of the 1991 ACM/IEEE Conference on Supercomputing, ser. SC, 1991, pp. 712-721.

[8] Y. Lee, R. Avizienis, A. Bishara, R. Xia, D. Lockhart, C. Batten, and K. Asanović, "Exploring the Tradeoffs Between Programmability and Efficiency in Data-parallel Accelerators," in Proceedings of the 38th Annual International Symposium on Computer Architecture, ser. ISCA, 2011, pp. 129-140.

[9] J. Gebis and D. Patterson, "Embracing and Extending 20th-Century Instruction Set Architectures," Computer, vol. 40, no. 4, pp. 68-75, 2007. 
[10] Intel $₫$ Architecture Instruction Set Extensions Programming Reference, Intel, March 2014.

[11] R. Espasa, M. Valero, and J. E. Smith, "Vector Architectures: Past, Present and Future," in Proceedings of the 12th International Conference on Supercomputing, ser. ICS, 1998, pp. 425-432.

[12] C. Lomont, "Introduction to Intel $₫$ Advanced Vector Extensions," Intel White Paper, 2011.

[13] R. M. Russell, "The CRAY-1 Computer System," Communications of the ACM, vol. 21, no. 1, pp. 63-72, Jan. 1978.

[14] T. Hayes, O. Palomar, O. Unsal, A. Cristal, and M. Valero, "Vector Extensions for Decision Support DBMS Acceleration," in Proceedings of the 2012 45th Annual IEEE/ACM International Symposium on Microarchitecture, ser. MICRO, 2012, pp. 166-176.

[15] G. Chrysos, "Intel $®$ Xeon Phi ${ }^{\mathrm{TM}}$ coprocessor (codename Knights Corner)," in Proceedings of the 24th Hot Chips Symposium, ser. HC, 2012.

[16] L. Seiler, D. Carmean, E. Sprangle, T. Forsyth, M. Abrash, P. Dubey, S. Junkins, A. Lake, J. Sugerman, R. Cavin, R. Espasa, E. Grochowski, T. Juan, and P. Hanrahan, "Larrabee: A Many-core x86 Architecture for Visual Computing," in ACM SIGGRAPH 2008 Papers, ser. SIGGRAPH ACM, 2008, pp. 18:1-18:15.

[17] R. Espasa, M. Valero, and J. E. Smith, "Out-of-Order Vector Architectures," in Proceedings of the 30th Annual ACM/IEEE International Symposium on Microarchitecture, ser. MICRO, 1997, pp. 160-170.

[18] D. Koch and J. Torresen, "FPGASort: a high performance sorting architecture exploiting run-time reconfiguration on FPGAs for large problem sorting," in Proceedings of the 19th ACM/SIGDA International Symposium on Field-Programmable Gate Arrays, ser. FPGA, 2011, pp. 45-54.

[19] R. Marcelino, H. Neto, and J. M. P. Cardoso, "Sorting Units for FPGABased Embedded Systems," in Distributed Embedded Systems: Design Middleware and Resources, ser. IFIP The International Federation for Information Processing, 2008, vol. 271, pp. 11-22.

[20] R. Mueller, J. Teubner, and G. Alonso, "Data processing on FPGAs" Proceedings of the VLDB Endowment, vol. 2, no. 1, pp. 910-921, 2009.

[21] N. Govindaraju, J. Gray, R. Kumar, and D. Manocha, "GPUTeraSort: high performance graphics co-processor sorting for large database management," in International Conference on Management of Data, ser. SIGMOD, 2006, pp. 325-336.

[22] D. Merrill and A. Grimshaw, "High Performance and Scalable Radix Sorting: A case study of implementing dynamic parallelism for GPU computing," Parallel Processing Letters, vol. 21, no. 02, pp. 245-272, 2011

[23] N. Satish, M. Harris, and M. Garland, "Designing efficient sorting algorithms for manycore GPUs," in International Symposium on Paralle \& Distributed Processing, ser. IPDPS, 2009, pp. 1-10.

[24] M. Pharr and W. R. Mark, "ispc: A SPMD Compiler for HighPerformance CPU Programming," in Innovative Parallel Computing, ser. InPar, 2012, pp. 1-13.

[25] C. Gregg and K. Hazelwood, "Where is the Data? Why You Canno Debate CPU vs. GPU Performance Without the Answer," in International Symposium on Performance Analysis of Systems and Software, ser. ISPASS, 2011, pp. 134-144.

[26] P. A. Boncz, M. Zukowski, and N. Nes, "MonetDB/X100: HyperPipelining Query Execution," in Biennial Conference on Innovative Data Systems Research, ser. CIDR, vol. 5, 2005, pp. 225-237.

[27] R. Cole, "Parallel Merge Sort," SIAM Journal on Computing, vol. 17, no. 4 , pp. $770-785,1988$

[28] D. Pasetto and A. Akhriev, "A comparative study of parallel sor algorithms," in Conference on Object-Oriented Programming Systems, Languages, and Applications, ser. OOPSLA, 2011, pp. 203-204.

[29] P. Tsigas and Y. Zhang, "A Simple, Fast Parallel Implementation of Quicksort and its Performance Evaluation on SUN Enterprise 10000," in Eleventh Euromicro Conference on Parallel, Distributed and NetworkBased Processing, 2003, pp. 372-381.

[30] V. W. Lee, C. Kim, J. Chhugani, M. Deisher, D. Kim, A. D. Nguyen, N. Satish, M. Smelyanskiy, S. Chennupaty, P. Hammarlund et al., "Debunking the 100X GPU vs. CPU myth: an evaluation of throughput computing on CPU and GPU," in ACM SIGARCH Computer Architecture News, vol. 38, no. 3, 2010, pp. 451-460.
[31] N. Satish, C. Kim, J. Chhugani, A. D. Nguyen, V. W. Lee, D. Kim, and P. Dubey, "Fast Sort on CPUs, GPUs and Intel MIC Architectures," Intel Labs, 2010.

[32] M. Yourst, "PTLsim: A Cycle Accurate Full System x86-64 Microarchitectural Simulator," in IEEE International Symposium on Performance Analysis of Systems Software, ser. ISPASS, 2007, pp. 23-34.

[33] Intel囚64 and IA-32 Architectures Optimization Reference Manual, Intel, March 2014

[34] P. Rosenfeld, E. Cooper-Balis, and B. Jacob, "DRAMSim2: A Cycle Accurate Memory System Simulator," IEEE Computer Architure Letters, vol. 10, no. 1, pp. 16-19, Jan. 2011.

[35] R. Espasa, F. Ardanaz, J. Emer, S. Felix, J. Gago, R. Gramunt, I. Hernandez, T. Juan, G. Lowney, M. Mattina, and A. Seznec, "Tarantula: A Vector Extension to the Alpha Architecture," in Proceedings of the 29th Annual International Symposium on Computer Architecture, ser. ISCA, 2002, pp. 281-292.

[36] F. Quintana, J. Corbal, R. Espasa, and M. Valero, "Adding a Vector Unit to a Superscalar Processor," in Proceedings of the 13th International Conference on Supercomputing, ser. ICS, 1999, pp. 1-10.

[37] D. Abadi, P. A. Boncz, S. Harizopoulos, S. Idreos, and S. Madden, "The design and implementation of modern column-oriented database systems," Foundations and Trends in Databases, vol. 5, no. 3, pp. 197280, 2013.

[38] G. P. Copeland and S. N. Khoshafian, "A Decomposition Storage Model," in Proceedings of the 1985 ACM SIGMOD International Conference on Management of Data, ser. SIGMOD, 1985, pp. 268279.

[39] R. Sedgewick, "Implementing Quicksort Programs," Communications of the ACM, vol. 21, no. 10, pp. 847-857, Oct. 1978.

[40] A. N. Habermann, "Parallel neighbor-sort (or the glory of the induction principle)," Carnegie Mellon University, Tech. Rep., 1972.

[41] K. E. Batcher, "Sorting networks and their applications," in Proceedings of the April 30-May 2, 1968, Spring Joint Computer Conference, ser AFIPS '68 (Spring), 1968, pp. 307-314.

[42] J. Fang, A. L. Varbanescu, H. Sips, L. Zhang, Y. Che, and C. Xu, "An Empirical Study of Intel Xeon Phi," arXiv preprint arXiv:1310.5842, 2013.

[43] D. E. Knuth, The Art of Computer Programming, Volume 3: Sorting and Searching (2nd Edition). Addison Wesley Longman Publishing Co., Inc., 1998.

[44] A. Gottlieb, R. Grishman, C. P. Kruskal, K. P. McAuliffe, L. Rudolph, and M. Snir, "The NYU Ultracomputer - Designing an MIMD Shared Memory Parallel Computer," IEEE Transactions on Computers, vol. 100, no. 2, pp. 175-189, 1983.

[45] J. Shun, G. E. Blelloch, J. T. Fineman, P. B. Gibbons, A. Kyrola, H. V. Simhadri, and K. Tangwongsan, "Brief Announcement: The Problem Based Benchmark Suite," in Proceedings of the 24th ACM Symposium on Parallelism in Algorithms and Architectures, ser. SPAA, 2012, pp. 68-70.

[46] G. E. Blelloch, J. T. Fineman, P. B. Gibbons, and J. Shun, "Internally Deterministic Parallel Algorithms Can Be Fast," in Proceedings of the 17th ACM SIGPLAN Symposium on Principles and Practice of Parallel Programming, ser. PPoPP, 2012, pp. 181-192.

[47] S. Li, K. Chen, J. H. Ahn, J. B. Brockman, and N. P. Jouppi, "CACTIP: Architecture-level modeling for SRAM-based structures with advanced leakage reduction techniques," in International Conference on Computer-Aided Design, ser. ICCAD, 2011, pp. 694-701.

[48] J. H. Ahn, M. Erez, and W. J. Dally, "Scatter-Add in Data Parallel Architectures," in Proceedings of the 11th International Symposium on High-Performance Computer Architecture, ser. HPCA, 2005, pp. 132142

[49] S. Kumar, D. Kim, M. Smelyanskiy, Y.-K. Chen, J. Chhugani, C. J. Hughes, C. Kim, V. W. Lee, and A. D. Nguyen, "Atomic Vector Operations on Chip Multiprocessors," in Proceedings of the 35th Annual International Symposium on Computer Architecture, ser. ISCA, 2008, pp. $441-452$. 